\title{
Modified Elite Chaotic Artificial Fish Swarm Algorithm for PAPR Reduction in OFDM Systems
}

\author{
Jie Zhou*†, Eryk Dutkiewicz*, Ren Ping Liu ${ }^{\ddagger}$, Xiaojing Huang ${ }^{\ddagger}$, Gengfa Fang*, Yuanan Liu ${ }^{\dagger}$ \\ * Department of Engineering, Macquarie University, Australia \\ ${ }^{\dagger}$ School of Electronics Engineering, Beijing University of Post and Telecommunication, Beijing, China \\ $\ddagger$ Commonwealth Scientific and Industrial Research Organisation (CSIRO), Australia \\ Email:jie.zhou8@students.mq.edu.au,\{eryk.dutkiewicz,Gengfa.Fang\}@mq.edu.au,ren.liu@csiro.au,yuliu@bupt.edu.cn
}

\begin{abstract}
Orthogonal frequency division multiplexing (OFD$M)$ is a leading technology in the field of broadband wireless communications. In OFDM systems, a high peak-to-average power ratio (PAPR) is a critical issue, which may cause a nonlinear distortion and reduce power efficiency. To reduce the PAPR, partial transmit sequences (PTS) technique can be applied to the transmit data. However, the phase factor sequence selection in PTS technique is a non-linear optimization problem and it suffers from high complexity and memory use when there is a large number of non-overlapping sub-blocks in one symbol. In this paper a novel modified elite chaotic artificial fish swarm algorithm for PTS method (MECAFSA-PTS) is proposed to generate the optimum phase factors. The MECAFSA-PTS method is evaluated with extensive simulations and its performance is compared with quantum evolutionary and selective mapping algorithms. Our results show that the proposed MECAFSA-PTS algorithm is efficient in PAPR reduction.
\end{abstract}

Index Terms-OFDM, PAPR, partial transmit sequence, artificial fish swarm algorithm.

\section{INTRODUCTION}

Recent advances in broadband mobile communication technologies expand the scope of applications of orthogonal frequency division multiplexing (OFDM) [1]. OFDM has been intensively studied and widely applied in many areas, such as Asymmetric Digital Subscriber Line (ADSL), Wireless Local Area Networks (WLAN), and Long Term Evolution (LTE). In OFDM systems, A high peak-to-average power ratio (PAPR) will not only reduce power efficiency but also destruct the orthogonality of subcarriers. Therefore, one of the major challenges in designing OFDM systems is PAPR reduction.

A number of signal scrambling and signal distortion techniques have been proposed for PAPR reduction, such as Selective Mapping (SLM), Partial Transmission Sequence (PTS), clipping, phase optimization, coding schemes, constellation shaping, etc. Amongst these methods, PTS is an effective technique for achieving low PAPR. The key to achieve such a goal lies in the phase factors selection algorithm, which must have a good balance between complexity and performance. However, in the phase factors selection, the computational complexity grows exponentially with the number of subcarriers, and the problem is NP hard. In this context, many methods have been proposed, including SLM, quantum evolutionary algorithms (QEA) and simulated annealing (SA) algorithms.
A PTS technical using the simulated annealing (SA) methods has been proposed in [2]. This algorithm was shown to perform well when the number of subcarriers is small. However, when the number of subcarriers is large, the rate of convergence is low and the algorithm can easily converge to a local optimum. An artificial bee colony algorithm for PTS technical (ABC-PTS) has been attempted in [3]. In their work the authors study a PTS with artificial bee colony. They also compare ABC-PTS with the particle swarm optimization (PSO) method. Another method called QEA-PTS based on the QEA has been used for the PTS method in [4]. The proposed method minimizes the PAPR using the knowledge related to quantum computing and evolutionary algorithms. However, the convergence speed is still low because of the sequential nature of the QEA operations. Moreover, QEA can easily fall into premature convergence, which makes it difficult to converge to the global optimum when the number of subcarriers is high.

Artificial fish swarm algorithm (AFSA) is a heuristic search algorithm based on animal social behavior and swarm intelligence, which was firstly proposed in literature [5] in 2001. As a novel evolutionary algorithm with easy implementation, AFSA shows many promising characteristics such as strong robustness and adaptive ability for many optimization problems. However, as each artificial fish (AF) has a limited vision, the traditional AFSA is easy to fall into a local optimum, which always leads to a premature and suboptimal solution. Moreover, due to the randomness of behaviors, the AF with best position in the swarm will not be recorded in traditional AFSA, which means the optimal solution may be lost during the iterative process. In this case, the convergence speed of the algorithm will become very slow.

In order to get to the global convergence, based on the traditional ASFA, this paper proposes a new modified elite chaotic artificial fish swarm algorithm for the PTS method (MECAFSA-PTS). Then MECAFSA-PTS is used for solve the PAPR reduction problem in OFDM systems. MECAFSAPTS has a fast global convergence rate and strong robustness, and its implementation is flexible.

This paper is organized as follows. In Section II, we describe the mathematical model and objective function. In Section III, we describe the implementation details of the MECAFSA-PTS 
method. In Section IV, both simulation results and discussion are presented. Finally, Section V gives the conclusions.

\section{System MODEL}

This section describes the system model of PTS selection with respect to the input symbol sequence. In previous work, Jung-Chieh et al formulated PTS selection as a multidimensional optimization problem [4], which is adopted in this work.

We assume that each OFDM symbol consists of $L$ subcarriers and the complex envelope of the transmission signal is given by:

$$
x_{n}=\frac{1}{\sqrt{L}} \sum_{l=0}^{L-1} Y_{l} e^{i 2 \pi n l\left(\frac{1}{L}\right)}
$$

where $n$ is the discrete time index, $i$ equal to $\sqrt{-1}$, and $Y=$ $\left[\begin{array}{llll}Y_{0} & Y_{1} & \cdots & Y_{L-1}\end{array}\right]$ is the input symbol sequence.

The peak-to-average power ratio can be written as:

$$
P A P R=10 \log _{10} \frac{\max \left\{\left|x_{n}\right|^{2}\right\}}{E\left\{\left|x_{n}\right|^{2}\right\}}
$$

where $E$ denotes the expected value operation.

In the OFDM system, we can divide the input data $Y=$ $\left[\begin{array}{llll}Y_{0} & Y_{1} & \cdots & Y_{L-1}\end{array}\right]$ in to $V$ non-overlapping sub-blocks $\left\{Y_{v}, v=0,1, \cdots, V-1\right\}$, which can be shown as

$$
Y=\sum_{v=0}^{V-1} Y_{v}
$$

Given a set of input data $Y$, our objective is to select an appropriate phase weighting sequence to minimize the PAPR. A phase weighting sequence can be expressed as a vector with length $V$, which can be represented as:

$$
D=\left[\begin{array}{llll}
d_{0} & d_{1} & \cdots & d_{V-1}
\end{array}\right]
$$

where $v \in[1, V-1]$, and $\left\{\varphi_{v}, v=0,1, \cdots, V-1\right\}$ is phase factors selected from the range $\varphi_{v} \in[0,2 \pi), d_{v}=\exp \left(j \varphi_{v}\right)$ is the phase weighting factor. In practice, the phase factors are select from a limited set, which can be represented as:

$$
\varphi_{v} \in\left\{e^{i 2 \pi \omega / W} \mid \omega=0,1, \cdots W-1\right\}
$$

where $W$ is the set of permitted phase factors. In this paper we only consider $\omega=0,1,2,3$, which means $d_{v} \in\{1, i,-1,-i\}$.

After selecting a proper phase weighting factor, it is multiplied by the input data to reduce the PAPR, which can be represented as:

$$
Y^{\prime}=Y \cdot D=\left[\begin{array}{llll}
d_{0} Y_{0} & d_{1} Y_{1} & \cdots & d_{V-1} Y_{V-1}
\end{array}\right]
$$

After being optimized by the phase weighting factor, the discrete time transmitted signal can be represented as $x_{n}{ }^{\prime}(D)$. The side information $D$ will be passed to the receiver through other channels.

So the objective function can be summarized as follows:
Minimize

$$
f(D)=\frac{\max \left\{\left|x_{n}{ }^{\prime}(D)\right|^{2}\right\}}{E\left\{\left|x_{n}^{\prime}(D)\right|^{2}\right\}}
$$

subject to

$$
\varphi_{v} \in\left\{e^{i 2 \pi \omega / W} \mid \omega=0,1, \cdots W-1\right\}
$$

If we want to minimize the fitness function $f(D)$, we must select each phase factor from the set $\varphi_{v} \in$ $\left\{e^{i 2 \pi \omega / W} \mid \omega=0,1, \cdots W-1\right\}$. Moreover, as changing a common angle on all sub-blocks cannot change PAPR, we can just consider the solution space in $W^{V-1}$ and ignore the value of $d_{0}$.

\section{PAPR REDUCTION BASED ON MECAFSA}

Inspired by the natural swarm behaviour of the fish, artificial fish swarm algorithm (AFSA) is an optimization method with similar features of the genetic algorithm such as the objective function and iterations. Based on swarm intelligence, it searches the global optimum in the solution space for complex nonlinear high dimensional problems based on different behaviours. In order to achieve the global optimum and to improve the convergence speed, in this section, we propose the new modified elite chaotic artificial fish swarm algorithm for PTS method (MECAFSA-PTS) to solve the PAPR reduction problem in OFDM systems.

\section{A. The principle of the traditional AFSA}

AFSA keeps a fish swarm with a fixed number of artificial fish (AF), and the position of each AF is a potential solution of the problem. The water area equals to the whole search space. The AF swims towards a position with more food in the water area iteratively, which means the movement is driven by nutrition. The concentration of food depends on the problem utility function, so the algorithm can achieve optimization by AF swarm searching behavior. The AF communicate with other AFs through behavior, which means information about concentrations of food spreads within the swarm. In AFSA, a pair of AF individuals has a distance value, which can be Hamming distance of two encoded AF. Each AF has a visual area value, which means the $\mathrm{AF}$ can only see and follow another $\mathrm{AF}$ when the distance between two $\mathrm{AF}$ is within a certain range. In nature, fish swim to the place with more nutrition, and usually gather in groups to avoid dangers and enemy. By detailed observation, the behavior of fish is abstracted as three typical behaviors: prey, swarm and follow.

\section{B. Representation of Artificial Fish and Swarm}

In MECAFSA-PTS, each AF individual is equivalent to a phase weighting sequence $D=\left[\begin{array}{llll}d_{1} & d_{2} & \cdots & d_{V-1}\end{array}\right]$. Each $\mathrm{AF}$ is encoded into a vector, where $V$ is the number of the non-overlapping sub-blocks in one symbol, $d_{v}$ is the $v_{t h}$ phase weighting factor of AF position, and each $d_{v}$ is selected from the set $1,-1, \mathrm{i},-\mathrm{i}, v \in[1, V-1]$. For example, when the number 
of non-overlapping sub-blocks $V=16$, a possible solution vector can be: $D=\left\{\begin{array}{llll}i & -i & \cdots & 1\end{array}\right\}_{1 \times 15}$.

Suppose that in the swarm there are $K$ AF individuals. The whole swarm can be represented as: $P_{A F}=\left\{\begin{array}{llll}D_{1} & D_{2} & \cdots & D_{K}\end{array}\right\}$. If there are more AF in the swarm, the MECAFSA-PTS convergence speed is faster, but the computational complexity will be higher.

The Logistic map was first proposed in [6], which can be expressed by

$$
x_{l+1}=4 x_{l}\left(1-x_{l}\right)
$$

where $x_{l}$ is a number between 0 and 1 . The Logistic map can generate chaotic numbers with low complexity, it has chaotic behaviour and non-linear characteristics.

MECAFSA-PTS uses (9) to generate the chaotic sequence, and then uses a simple map to generate each initial AF, which can be shown as:

$$
d_{v}=\left\{\begin{array}{lr}
1 & 0<x_{v}<0.25 \\
-1 & 0.25 \leq x_{v}<0.5 \\
i & 0.5 \leq x_{v}<0.75 \\
-i & 0.75 \leq x_{v}<1
\end{array} .\right.
$$

Before the iteration starts, the iteration counter is set to zero.

\section{Distance, Visual area and Concentration Factor}

The distance between two AF individuals $\left\{D_{i}, D_{j}\right\}$ is denoted as $d i s_{i j}=\left\|D_{i}-D_{j}\right\|$, where $\left\|D_{i}-D_{j}\right\|$ denotes the Hamming distance between the two AF vectors. The visual area $V I S_{i}$ represents the vision distance of $i_{t h} \mathrm{AF}$ individual, which means $D_{i}$ cannot see $D_{j}$ if the Hamming distance $\left\|D_{i}-D_{j}\right\|>V_{i}$. Let $n_{i}$ represent the number of AF individuals within the visual area of $D_{i}$, and $n_{\text {total }}$ represent the total number of AF individuals in the whole swarm. If the ratio of $\frac{n_{i}}{n_{\text {total }}} \geq \delta$, it means the area is overcrowded, $D_{i}$ will get away from this area and prey for other food.

\section{Objective Function}

The objective function of MECAFSA-PTS can be represented as the food concentration, and the aim of the algorithm is to find the position with the highest food concentration with AF individuals. As we need to minimize the PAPR, the objective function of MECAFSA-PTS can be show as $-f(D)$, and all parameters have same meaning with that in (7). According to (7), the PAPR has an inverse relationship with the food concentration. So when the AF get to the position with highest food concentration, the corresponding phase weighting sequence has the lowest PAPR. As most AF in the swarm swim to a better position iteratively, they finally get the optimal phase weighting sequence.

\section{E. Elite list of Artificial Fish}

As the iteration characteristic of MECAFSA-PTS, we set a elite list to record the best AF in position with the highest food concentration. In another word, the corresponding phase weighting sequence with the lowest PAPR is recorded. After all AF individuals move to the new position in each iteration, we will compare the best AF in the swarm with the individual in elite list. If the best AF has a lower PAPR than the individual in elite list, we will update the elite list with the best AF.

\section{F. Behaviors of Artificial Fish}

There are four behaviors in MECAFSA-PTS, follow behavior, swarm behavior, prey behavior and random behavior. Unlike the traditional AFSA, each behavior has a fixed priority in MECAFSA-PTS. The follow behavior has the highest priority, next the swarm behavior, followed by the prey behavior. The random behavior has the lowest priority. Based on the principles in section $\mathrm{A}$, we can describe the behaviors of MECAFSA-PTS as follow:

1) Follow behavior: The follow behavior of $i_{t h}$ individual $D_{i}$ will be executed, if two constraints are satisfied.

Constraint 1: The ratio of $\frac{n_{i}}{n_{\text {total }}}<\delta$, where $n_{i}$ is the number of AF individuals within the visual area of $D_{i}, n_{\text {total }}$ is the total number of AF individuals in the whole swarm, and is the crowd factor.

Constraint 2: Within the visual area of $D_{i}$, at least one artificial fish has a lower PAPR than $D_{i}$.

If the above two constraints are met, $D_{i}$ will follow and move to an individual $D_{j}$ within the visual area. $D_{j}$ must have a lower PAPR than $D_{i}$. If more than one individual meet the constraints, $D_{i}$ will select a random one amongst them. If any constraint is not satisfied, $D_{i}$ will try the swarm behavior. Different from the traditional AFSA, the prey behavior will not be executed immediately if the constraint is not satisfied.

In each iteration, there is a maximum limit of moving steps $S_{\max }$. First we calculate vector $D_{s u b}$ by using $D_{j}-D_{i}$, then a binary sequence rand is multiplied with vector $D_{s u b}$. In binary sequence $r a n d$, there are $S_{\max }$ elements equal to 1 , and ( $V-$ $\left.1-S_{\max }\right)$ elements equal to 0 , where $V$ is the number of nonoverlapping sub-blocks in one OFDM symbol. The positions of both 1 and 0 elements are random in the binary sequence. In this way, the number of different elements between $D_{i}$ and $D_{j}$ is smaller than $S_{\max }$, and $D_{i}$ swims toward a better artificial fish $D_{j}$. This process can be shown as:

$$
\begin{gathered}
D_{s u b}=\operatorname{rand} \cdot\left(D_{j}-D_{i}\right) \\
D_{i}^{\text {new }}=D_{i}+D_{s u b}
\end{gathered}
$$

where the operator - is the point multiplication operation.

2) Swarm behavior: If any constraint of the follow behavior is not satisfied, the $i_{t h}$ individual $D_{i}$ will try the prey behavior. The swarm behavior will carry out if the following two constraints are satisfied:

Constraint 1: The ratio $\frac{n_{i}}{n_{\text {total }}}<\delta$, all the parameters have the same meaning as in section 1).

Constraint 2: There are at least two artificial fish within the visual area of $D_{i}$.

If the above two constraints are satisfied, we calculate the total number of neighborhoods within the visual area, calculate 
the center position of them, and let $D_{i}$ swim to the center position.

Assume there are $N_{b}$ neighborhoods within the visual area, the $k_{t h}$ neighborhood $D_{k}$ can be represented as a vector $D_{k}=\left\{\begin{array}{lllll}d_{1}^{k} & \cdots & d_{v}^{k} & \cdots & d_{V-1}^{k}\end{array}\right\}_{1 \times(V-1)}$, and each $a_{l}^{k}$ is selected from the set $1,-1, \mathrm{i},-\mathrm{i}$. The center position of neighborhoods $D_{\text {certer }}$ can be calculated as:

$$
D_{\text {certer }}=f\left(\frac{1}{N_{b}} \sum_{k=1}^{N_{b}} D_{k}\right)
$$

where $k$ is the index of the neighborhoods, and $D_{\text {certer }}$ is the center position of the neighborhoods. Assume $D_{\text {center }}=\left[\begin{array}{lllll}d_{1}{ }^{\prime} & \cdots & d_{v}{ }^{\prime} & \cdots & d_{V-1}{ }^{\prime}\end{array}\right]$, we need to use a simple map function $f()$ to fix the position:

$d_{v}{ }^{\prime}=f\left(\frac{1}{N_{b}} \sum_{k=1}^{N_{b}} d_{v}^{k}\right)=\left\{\begin{array}{cl}1 & \frac{\pi}{4} \leq \arg \left(\frac{1}{N_{b}} \sum_{k=1}^{N_{b}} d_{v}^{k}\right)-<\frac{\pi}{4} \\ i & \frac{3 \pi}{4} \leq \arg \left(\frac{1}{N_{b}} \sum_{k=1}^{N_{b}} d_{v}^{k}\right)<\frac{\pi}{4} \\ -1 & \frac{5 \pi}{4} \leq \arg \left(\frac{1}{N_{b}} \sum_{k=1}^{N_{b}} d_{v}^{k}\right)<\frac{3 \pi}{4} \\ -i & \frac{7 \pi}{4} \leq \arg \left(\frac{1}{N_{b}} \sum_{k=1}^{N_{b}} d_{v}^{k}\right)<\frac{5 \pi}{4}\end{array}\right.$

where $\arg ()$ is the phase angle function. To facilitate the representation, the phase angle is limited to the range $\left(-\frac{\pi}{4}, \frac{7 \pi}{4}\right]$ instead of the traditional range $(-\pi, \pi]$. In this way, we get a new individual $D_{\text {certer }}$. Then MECAFSA-PTS updates $D_{i}$ by (15) and (16):

$$
\begin{gathered}
D_{\text {sub }}=\operatorname{seq} \cdot\left(D_{\text {certer }}-D_{i}\right) \\
D_{i}^{\text {new }}=D_{i}+D_{\text {sub }}^{2}
\end{gathered}
$$

All the parameters are same as (11) and (12).

3) Prey Behavior: If any constraint of the swarm behavior is not satisfied, the $i_{t h}$ individual $D_{i}$ will try the prey behavior. First a random artificial fish is generated within the visual area of $D_{i}$. Specifically, MECAFSA-PTS generates an individual $D_{j}$, and makes sure the Hamming distance $\left\|D_{i}-D_{j}\right\| \leq$ $V I S_{i}$, where $V I S_{i}$ is the visual distance of $i_{t h}$ AF individual $D_{i}$. Firstly we select random $V I S_{i}$ positions on vector $D_{i}$, and generate $V I S_{i}$ numbers between 0 and 1 with the Logistic map as (9). Then we change the value on these positions on vector $D_{i}$ into the set $1,-1, \mathrm{i},-\mathrm{i}$ with a simple map (10). In this way, a new artificial fish $D_{j}$ is generated. Then we calculate the PAPR of $D_{j}$. If the PAPR of $D_{j}$ is lower than $D_{i}, D_{i}$ is moved toward to $D_{j}$ with (11) and (12).

If the PAPR of $D_{j}$ is higher than $D_{i}$, we generate another new AF with the above steps in the prey behavior. If it still cannot find an AF with a lower PAPR after the maximum number of times, the random behavior will be carried out.
4) Random Behavior: Generate $V-1$ numbers between 0 and 1 with the Logistic map as (9), then change the value into the set $1,-1, \mathrm{i},-\mathrm{i}$ with the simple map (10). In this way, a new artificial fish $D_{j}$ is generated. This $D_{j}$ can be either in the visual area of $D_{i}$ or outside. Then $D_{i}$ will swim toward to $D_{j}$ with (11) and (12) regardless of the PAPR value of $D_{j}$.

\section{G. Stopping Condition}

Repeat four behaviors according to the priority order until all AF in the swarm moved to another position. After that update the elite list with the best AF in the swarm, and check whether the algorithm has reached the maximum number of iterations. If the algorithm has reached the maximum number of iterations, end the algorithm and output the best AF on the elite list as the result with the lowest PAPR. If algorithm has not reached the maximum number of iterations, start the next iteration and add one to the iteration counter.

\section{EXPERIMENTAL STUDY}

In this section, we present the simulation results with different algorithms for the PTS selection problem. To demonstrate the effectiveness of the algorithms, we use the complementary cumulative distribution function (CCDF) to evaluate the merits of the algorithms. The CCDF function is defined as:

$$
C C D F=P\left\{P A P R>P A P R_{0}\right\}
$$

where $P$ is the probability function.

The Matlab is used as the programming language in simulation. The objective function of the SLM selection problem has been computed using (7). In the simulations, the input symbol sequence is considered uniformly distributed among all the symbols and the all the symbols are modulated with QPSK. In the following experiments, we set the number of symbols to $1 \times 10^{3}$. We also set the maximum number of iterations of both QEA-PTS and MECAFSA-PTS to 20. For the purpose of comparison, the 8 point SLM and the original simulation curve are also presented. In order to fairly compare algorithms with different numbers of subcarriers, the number of subcarriers are set to 128 . The same population size $P o p=40$ has been considered for both MECAFSA-PTS and QEA-PTS.

Fig. 1, Fig. 2 show the results for MECAFSA-PTS, QEAPTS and SLM to select the phase weighting factor for the PAPR reduction problem with the phase weighting sequence length equal to 4 and 8 respectively with the above parameter settings. The maximum iterations are set to 20 for each algorithm. We set the number of subcarriers to 128 . The figures also include the results for the CCDF with no PTS (referred to as original in the figures).

As Fig. 1 shows, MECAFSA-PTS yields much better results compared to QEA-PTS, SLM and the original. For example, when the phase weighting sequence length is 4 and $C C D F=$ $10^{-3}$, the PAPR0 for MECAFSA-PTS is around $6.9 \mathrm{~dB}$, while the result for QEA, SLM and original are $7.8 \mathrm{~dB}, 7.3 \mathrm{~dB}$ and $9.9 \mathrm{~dB}$ respectively. In Fig. 2, we can get similar conclusions 


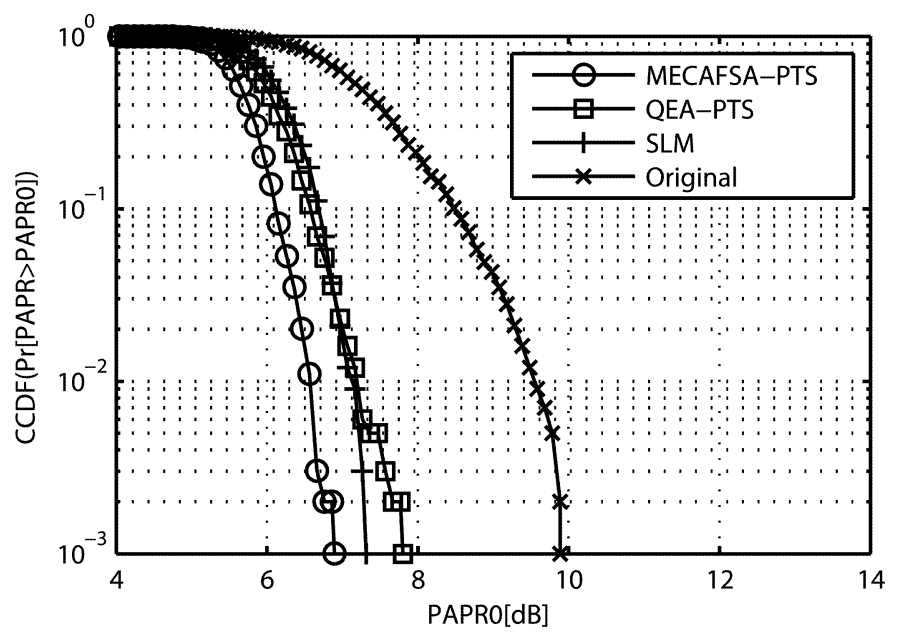

Fig. 1. CCDF of the PAPR with $\mathrm{V}=4$, subcarrier=128.

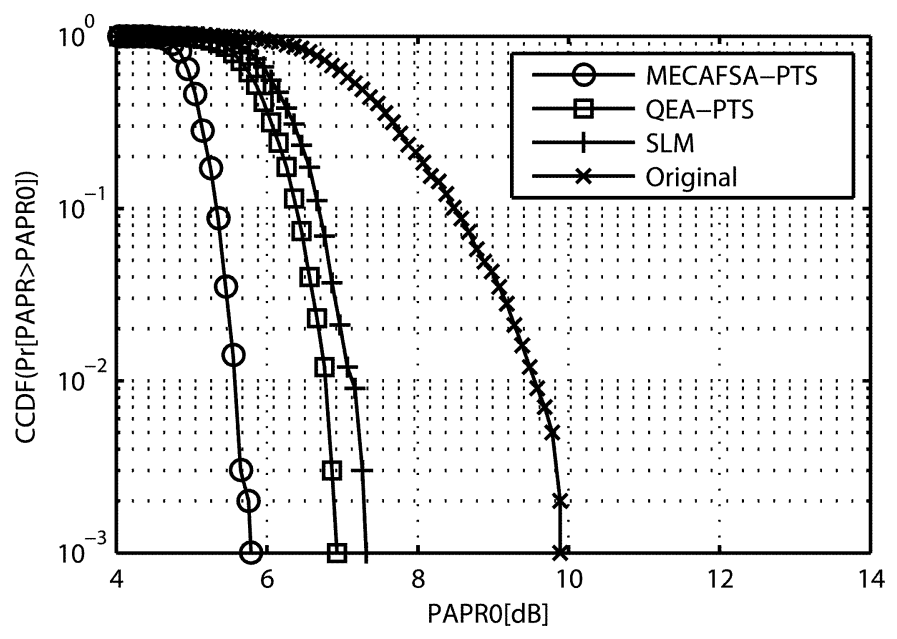

Fig. 2. CCDF of the PAPR with $V=8$, subcarrier $=128$.

that the reduction of the PAPR for MECAFSA-PTS is $1 \mathrm{~dB}$ or higher compared to the other algorithms when the number of subcarriers is set to 128 .

Fig. 3 illustrates the convergence of the mean PAPR value of MECAFSA-PTS and QEA-PTS during the 50 iterations. As it can be observed in Fig. 3, MECAFSA-PTS has shown better results when compared with QEA-PTS approach. At the initial 20 iterations, the mean PAPR of both algorithms decrease. After that QEA-PTS displayed premature convergence, which means the QEA-PTS gets stuck in local minima that are hard to escape. On the other hand, it can be seen that MECAFSAPTS produces lower PAPR results much faster than QEA-PTS. It displays no premature convergence during the entire 50 iterations.

\section{CONClusion}

This paper proposes a new Modified Elite Chaotic Artificial Fish Swarm Algorithm (MECAFSA) for optimizing the

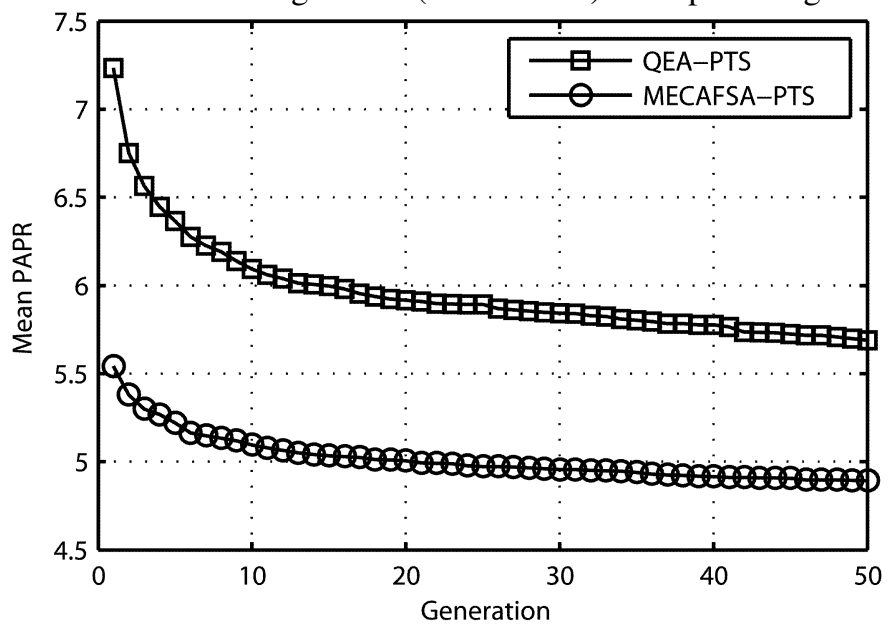

Fig. 3. Average PAPR change by generation with $V=8$, subcarrier $=128$.

PAPR in OFDM systems using a phase factors selection. An objective function is designed to evaluate the algorithm and simulations are performed to compare its performance with algorithms based on quantum evolutionary and selective mapping approaches. The results demonstrate the effectiveness of the proposed algorithm.

\section{ACKNOWLEDGMENT}

Jie Zhou is a recipient of a Macquarie University Research Excellence Scholarship and is under a cotutelle $\mathrm{PhD}$ arrangement between Macquarie University and Beijing University of Posts and Telecommunications. This work was supported in part by the National Natural Science Foundation of China (No.61170275), the National Science and Technology Major Project (No.2012ZX03001001-002), and the research project of Guangdong (No.2011B090400433).

\section{REFERENCES}

[1] J. Passerieux, F. Socheleau and C. Laot, "Achievable Rates over Doubly Selective Rician-Fading Channels under Peak-Power Constraint," IEEE Trans. Wireless Commun., vol. 12, pp. 586-594, 2013.

[2] T. Jiang, W. Xiang, P. C. Richardson, J. Guo and G. Zhu, "PAPR Reduction of OFDM Signals Using Partial Transmit Sequences With Low Computational Complexity," IEEE Trans. Broadcast., vol. 53, pp. 719-724, 2007.

[3] Y. Wang, W. Chen and C. Tellambura. "A PAPR reduction method based on artificial bee colony algorithm for OFDM signals," IEEE Trans. Wireless Commun., vol 9, pp. 2994-2999. 2010.

[4] J. Chen and C. Wen, "A Low-Complexity Scheme to Reduce the PAPR of an OFDM Signal Using Sign-Selection Algorithms," IEEE Signal Processing Letters., vol. 17, pp. 189-192, 2010.

[5] L.X. Li, Z.J. Shao and J.X. Qian, "An optimizing method based on autonomous animals: fish-swarm algorithm," Systems Engineering theory practice., vol. 22, pp.32-38,2002.

[6] R. M. May, "Simple mathematical models with very complicated dynamics," Nuture, vol. 261, pp. 459-467, 1976. 